\title{
Gestión educativa y desempeño. Estudio correlacional en la provincia de Recuay, Perú
}

\author{
Educational management and performance. Correlational study in the province \\ of Recuay, Perú
}

\author{
René GABRIEL YÉPEZ HuAMÁN ${ }^{1}$ \\ reneyepez1974@gmail.com \\ https://orcid.org/0000-0001-8186-0579 \\ Unidad de Gestión Educativa Local de Recuay, Perú
}

\section{RESUMEN:}

El presente estudio tiene como propósito identificar las características de la gestión educativa y el desempeño docente, mediante la cual el personal directivo realiza diferentes acciones destinadas para que el profesor realice sesiones de aprendizaje de calidad para lograr aprendizajes significativos en los estudiantes. En dicho contexto, surge la siguiente pregunta ¿hasta qué punto la gestión educativa se relaciona con la mejora del desempeño docente? Metodológicamente, el estudio posee alcance correlacional. Se llegó a concluir que las actividades pedagógicas de la plana directiva ejercen una influencia positiva para la mejora del desempeño docente.

\section{ABSTRACT:}

The purpose of this study is to identify the characteristics of educational management and teaching performance, through which the directive personnel carry out different actions aimed at allowing the teacher to carry out quality learning sessions to achieve meaningful learning in students. In this context, the following question arises, to what extent is educational management related to improving teacher performance? Methodologically, the study has a correlational scope. It was concluded that the pedagogical activities of the board of directors exert a positive influence for the improvement of teaching performance.Keywords: education management, pedagogical management, administrative management, institutional management, teaching performance.

\section{Palabras Clave:}

Gestión educativa; gestión administrativa; gestión institucional; desempeño docente.

\section{KEYWORDS:}

Educational management; administrative management; institutional management; teaching performance.

Recibido: 15/10/2021 - Aceptado: 20/11/2021 - Publicado: 23/12/2021

\footnotetext{
(C) Los autores. Este artículo es publicado por la Revista peruana de investigación e innovación educativa de la Facultad de Educación, Universidad Nacional Mayor de San Marcos. Este es un artículo de acceso abierto, distribuido bajo los términos de la licencia Creative Commons Atribución 4.0 Internacional (CC BY 4.0) [https://creativecommons.org/licenses/by/4.0/deed.es] que permite el uso, distribución y reproducción en cualquier medio, siempre que la obra original sea debidamente citada de su fuente original.
} 


\section{Introducción}

Se entiende por gestión educativa a las diversas actividades que realiza la plana jerárquica en la dirección de una escuela, siendo la primera obligación del director influir de manera positiva en la forma en que los docentes realizan los diversos procesos de enseñanza aprendizaje. Entonces, la gestión debe centrarse en propósitos pedagógicos, las otras actividades institucionales y administrativas, y deben favorecen la acción educativa del maestro, poniendo a disposición todos los recursos de una escuela para el logro de los aprendizajes de los estudiantes y su bienestar emocional.

De acuerdo con Alvarado (2018), la gestión educativa se divide por la amplitud y complejidad burocrática del órgano central del Ministerio, tal como se observa en el organigrama del viceministerio de gestión pedagógica. Ello comprende todas las actividades referidas al desarrollo educativo de los estudiantes, que finalmente se materializan en las instituciones educativas; la administrativa, que se relaciona con el manejo de los recursos que hacen posible el proceso educativo; y la institucional, con diversas actividades o procesos que son inherentes a la administración.

La gestión educativa se dimensiona en actividades pedagógicas que está conformado por la forma en que la dirección planifica y ejecuta acciones que aseguren el aprendizaje y la sana convivencia en el alumnado; actividades institucionales que está vinculada con los documentos de gestión, donde se diseña un diagnóstico institucional, se plantean metas y organizan actividades pedagógicas distribuidas en el año académico escolar; y las actividades administrativas que explican los lineamientos del buen manejo de los recursos económicos otorgados por el Ministerio de Educación.

Bush (2003) refiere que la gestión educativa es un campo de estudio y práctica sobre el funcionamiento de las organizaciones educativas. La gestión educativa tiene que estar preocupada centralmente con el propósito u objetivos de la educación. Estos propósitos o metas nos proporcionan el sentido fundamental de la dirección.

La gestión debe centrarse en temas educativos, en base a ciertos indicadores; como los resultados de las pruebas censales nacionales, el promedio de notas de las diversas áreas curriculares, el número de estudiantes que repite anualmente, el porcentaje de abandono y deserción escolar, la evolución de la matrícula en los últimos años, y la percepción de la violencia escolar por parte de los estudiantes se construye el diagnóstico pedagógico. Las acciones de una escuela están encaminadas a la disminución o aumento de los distintos indicadores pedagógicos, por ejemplo: el aumento del nivel satisfactorio en las pruebas de la evaluación censal, el promedio de notas de las diversas áreas curriculares, entre otros. Asimismo, que todos los estudiantes obtengan mejores calificaciones está en directa relación con el desarrollo de sus capacidades de reflexión y criticidad, de resolución de problema, de creatividad e innovación y la autoconfianza para enfrentar diversas evaluaciones y dificultades.

El plan de acompańamiento y monitoreo se realiza con el fin de mejorar el desempeño docente, para lo cual, el director debe monitorear y acompañar los procesos de planificación curricular, la actuación del docente en clase, el buen manejo de la convivencia escolar y las diferentes formas evaluativas. La planificación curricular es de gran importancia, es el momento antes que se inicien las clases, la etapa en la cual se seleccionan las competencias que se intenta desarrollar en el alumnado, el producto que se espera lograr y la forma en que se va a evaluar, con esto se evita la improvisación.

Asimismo, el director debe estar presente en las sesiones de aprendizaje con el fin de observar la actuación del docente y las relaciones de convivencia que se da en el alumnado, mediante una ficha de observación realiza apuntes de lo ocurrido en la clase, a fin de que al finalizar esta, en compañía del docente analizan y reflexionan las fortalezas y debilidades de la clase a fin de plantear actividades para su mejora. La observancia de las relaciones de convivencia en el alumnado es fundamental para identificar hechos de violencia escolar o maltrato entre los alumnos, de ser el caso la dirección debe intervenir y brindar el soporto emocional a través del departamento de sicología. La acción evaluativa debe ser considerada parte del proceso enseñanza 
aprendizaje, porque nos indica si realmente se desarrollaron las competencias en el alumnado, de no ser el caso el profesor está obligado a incidir en aquellos puntos más difíciles de entender. Y, también implica que los productos que se exigen pueden ser devueltos a los alumnos, a fin de que tengan la oportunidad de levantar algunas observaciones.

Estas actividades se construyen con la participación de todo el equipo docente y se plantea un cronograma de monitoreo de todos los profesores a lo largo del ańo académico escolar, también se implementan capacitaciones y el desarrollo de grupos de interaprendizaje donde los maestros comparten sus experiencias exitosas y distintos proyectos de innovación.

Cejas (2009) menciona que la gestión en educación trae consigo los procesos de planificación estratégica, así como considerar el entorno y las capacidades de la misma institución educativa.

La planificación estratégica se evidencia a través de los diferentes documentos de gestión donde se establecen los propósitos y propone diversas actividades para alcanzarlos. El primer documento de gestión se denomina Proyecto Educativo Institucional, donde se plantea la visión y misión de la escuela, se construye el diagnóstico institucional, se plantean actividades y se construyen indicadores a fin de evaluar la gestión, con el fin de tomar medidas de remediación. El segundo instrumento se denomina Plan Anual de Trabajo, en el cual se organizan todas las actividades a realizar en un ańo escolar en directa relación con las metas propuestas en el Proyecto Educativo Institucional. Ambos instrumentos son fundamentales para la organización de las actividades pedagógicas las cuales deben ser lideradas por el director. El tercer documento de gestión es el reglamento interno, en la cual se establecen los derechos y deberes de la comunidad educativa, señalando las obligaciones de cada individuo y su responsabilidad en el cuidado de los bienes institucionales.

Lograr las metas educativas está en relación con la acción de toda la comunidad educativa, la cual comparte la misma visión de la institución educativa que está dispuesta a trabajar en equipo con el fin de lograr la mejora educativa en las diversas áreas de trabajo. Por otro lado, un grupo humano que se encuentra en una ruptura de relaciones humanas, donde sólo se dan denuncias permanentes, traerá la desconfianza y el incumplimiento de los propósitos educativos.

El liderazgo de un director se observa en la medida que logra influenciar para que su personal docente alcance las metas educativas propuestas en los diferentes documentos de gestión. Un director demuestra su liderazgo en la medida que se actualiza de forma permanente en temas pedagógicos, mantiene un control emocional permanente en cualquier momento, plantea argumentos para movilizar a la comunidad educativa a la mejora permanente, muestra compasión ante la problemática emocional del alumnado y toma de forma inmediata medidas de remediación. El liderazgo de la gestión está relacionado con un empoderamiento académico, una inteligencia emocional y una conducta basada en valores, que mantiene aparte los temas personales del mundo laboral.

\section{Desempeño Docente}

Por otro lado, Martínez - Chairez (2016) explica que el desempeño docente desde una perspectiva de desarrollo profesional, es crucial para la mejora del aprendizaje significativo, el ciclo de supervisión en sus tres fases, considerando los momentos anteriores y posteriores a la observación, parecen ser determinantes para fomentar el desarrollo profesional. El desempeño se puede dimensionar en: preparación y organización de las actividades escolares, conducción de las actividades de enseñanza, relaciones pedagógicas con los estudiantes y evaluación de los procesos de aprendizaje.

\section{Preparación y organización de las actividades escolares}

La preparación de las actividades escolares es una acción previa al desarrollo de la clase en sí misma. El docente realiza su planificación anual, sus unidades didácticas y sus sesiones de aprendizaje, los cuales 
sustentan su actividad pedagógica en el aula. Esta considera desde la forma en que va a motivar a sus alumnos, recoger sus saberes previos, realizar las actividades para desarrollar los procesos cognitivos, el producto que van a desarrollar los estudiantes y la forma en que se va a realizar la evaluación. Ninguna sesión de aprendizaje debe realizarse en una escuela, sin que el profesor antes la haya planificado por lo tanto el instrumento más confiable es la sesión de aprendizaje, en la cual se plantean la competencia que se va a trabajar en la sesión, las actividades, la forma en que se va a evaluar y los enfoques transversales establecidos. Ello no implicaría que algunas acciones planificadas puedan ser variadas ante la circunstancia misma de la clase, dentro de una sesión ocurren hay veces situaciones inesperadas que el profesor debe tomarla y analizarla desde el punto de vista pedagógico (Vásquez-Rodríguez, 2010, López, Fuentes y Sánchez, 2021; Gonzáles et al. , 2021).

\section{Conducción de las actividades de enseñanza}

Gestionar las actividades de enseñanza se entiende como la forma en que el profesor enseña y se manifiesta en cómo se comunica, sus gestos, su tono de voz y la aplicación de la estrategia pedagógica planificada con anterioridad. El docente utiliza todos los medios que tiene a su disposición con el fin de lograr los aprendizajes en los estudiantes. En este proceso se asegura de forma permanente, si realmente, los estudiantes vienen aprendiendo la lección planificada a través de la retroalimentación.

El docente mediante la realización de preguntas se asegura que los estudiantes aprenden la lección, si realmente adquieren las competencias. En el caso que las respuestas de los estudiantes sean limitadas, entonces el maestro vuelve a repetir la lección con el fin de asegurar los aprendizajes.

El maestro debe fomentar el diálogo y el debate alturado de las ideas en su sesión con el fin de estimular el aprendizaje. Y esta debe considerar la exposición del maestro, la opinión del estudiante, el debate entre estudiantes, la colaboración entre los estudiantes para la creación de un producto de aprendizaje, en esencia la acción humana debe ser pedagógicamente dinámica en la que se fomenta el trabajo colaborativo.

\section{Relaciones pedagógicas con los estudiantes}

Las buenas relaciones humanas son parte fundamental para lograr aprendizajes de calidad, se debe regular una conducta adecuada donde se evite la violencia escolar y situación de humillación del alumnado. García y Ferreira (2005) consideran que uno de los objetivos de cada docente es lograr que la clase funcione adecuadamente, expresándose a través del cumplimiento de la calendarización, que traen inmersos el aprendizaje de contenidos, destrezas y socialización de los mismos estudiantes. La buena convivencia en un aula es clave para el desarrollo de la autoconfianza en el estudiante, en un ambiente de respeto y de relaciones sociales correctas donde se desarrolla la compasión y el trato igualitario a los alumnos se genera un ambiente propicio para los aprendizajes.

\section{Evaluación de los procesos de aprendizaje}

Marín-González explican (2020) que la evaluación diagnóstica o exploratoria posee como finalidad "evaluar las características que los estudiantes traen al proceso de enseñanza, es decir, sus conocimientos previos, los cuales se relacionan directamente con el aprendizaje" (p. 225). Mediante la evaluación identificamos el nivel de desarrollo de las competencias en los estudiantes que es útil para fomentar la reflexión en el propio alumno a fin de que realice las acciones personales para mejorar sus aprendizajes.

Finalmente, la gestión educativa de un director debe centrarse en lo pedagógico, y para ello, debe ejercer un liderazgo a fin de influenciar a la comunidad educativa a realizar acciones constantes para mejorar el desempeño de los docentes en las sesiones de aprendizaje. Además, es significativo que el director haya construido su plan de acompańamiento y monitoreo docente con la participación de todo su equipo de maestros y las diversas acciones se encuentren debidamente planificadas y cronogramadas. 
El buen desempeńo docente es básico para el logro de aprendizajes de calidad, esto se inicia desde la preparación de las sesiones de aprendizaje, la forma en que el docente ejecuta la sesión de aprendizaje, el buen manejo de la convivencia y la utilización de la evaluación para identificar debilidades, las cuales pueden ser superadas mediante acciones de retroalimentación.

En este sentido, se trata de evitar que la gestión educativa se centre en acciones administrativas, que no se les dé la importancia a las acciones pedagógicas y que no se realice de forma permanente el monitoreo del director a los docentes. Por otra parte, el aprendizaje de los alumnos se vería disminuido ante la improvisación de una clase, la ocurrencia de violencia escolar o procesos de evaluación que no desarrollen acciones de reflexión o retroalimentación.

\section{Método}

Metodológicamente, el presente estudio tiene un alcance correlacional, cuya finalidad es medir la relación entre la variable Gestión Educativa y Desempeño Docente. Según Hernández et al. (2014) los estudios que miden una correlación tienen como propósito el identificar la posibilidad de que, estadísticamente, exista una evidencia empírica que involucre a dos o más variables de estudio. Con el fin de medir las variables mencionadas, el presente estudio cuantifica las variables con el fin de establecer vinculaciones, que serán sometidas a pruebas estadísticas.

\section{Resultados}

Interpretación: De la tabla 1, se observa que la correlación Pearson es de 0.895 y la significación es inferior a 0.005 .

Tabla 1

Correlación entre Gestión Educativa y Desempeño Docente

\begin{tabular}{llcc} 
& & Ges.Educ & Des.Docen \\
\hline \multirow{3}{*}{ Ges.Educ } & Correlación (Pearson) & 1 &, $895^{* *}$ \\
& Signif. (bilateral) & &, 000 \\
& $\mathrm{~N}$ & 28 & 28 \\
\multirow{3}{*}{ Des.Docen } & Correlación (Pearson) &, $895^{* *}$ & 1 \\
& Signif. (bilateral) &, 000 & \\
& $\mathrm{~N}$ & 28 & 28 \\
\hline
\end{tabular}

Nota. ${ }^{* *}$ La correlación es significativa en el nivel 0,01 (bilateral).

Interpretación: De la tabla 2, se observa que la correlación Pearson es de 0.719 y la significación es inferior a 0.005 .

\section{Tabla 2}

Correlación Gestión Pedagógica y Desempeño Docente

\begin{tabular}{llcc} 
& & Ges. Peda & Des.Docen \\
\hline \multirow{3}{*}{ Ges.Peda } & Correlación (Pearson) & 1 &, $719^{* *}$ \\
& Signif. (bilateral) & &, 000 \\
& $\mathrm{~N}$ & 28 & 28 \\
\multirow{3}{*}{ Des.Docen } & Correlación (Pearson) &, $719^{* *}$ & 1 \\
& Signif. (bilateral) &, 000 & \\
& $\mathrm{~N}$ & 28 & 28 \\
\hline
\end{tabular}

Nota. ${ }^{* *}$ La correlación es significativa en el nivel 0,01 (bilateral).

Interpretación: De la tabla 3, se observa que la correlación Pearson es de 0.733 y la significación es inferior a 0.005 . 
Tabla 3

Correlación Gestión Institucional y Desempeño docente

\begin{tabular}{llcc} 
& & Ges.Insti & Des.Docen \\
\cline { 3 - 4 } Ges.Insti & Correlación (Pearson) & 1 &, $733^{* *}$ \\
& Signif. (bilateral) & &, 000 \\
& $\mathrm{~N}$ & 28 & 28 \\
Des.Docen & Correlación (Pearson) &, $733^{* *}$ & 1 \\
& Signif. (bilateral) &, 000 & 28
\end{tabular}

Nota. ${ }^{* *}$ La correlación es significativa en el nivel 0,01 (bilateral).

Interpretación: De la tabla 4, se observa que la correlación Pearson es de 0.780 y la significación es inferior a 0.005 .

Tabla 4

Correlación Gestión Administrativa y Desempeño Docente

\begin{tabular}{llcc} 
& & Ges.Ad & Des.Docen \\
\hline \multirow{3}{*}{ Ges.Ad } & Correlación (Pearson) & 1 &, $780^{* *}$ \\
& Signif. (bilateral) & &, 000 \\
& $\mathrm{~N}$ & 28 & 28 \\
\multirow{3}{*}{ Des.Docen } & Correlación (Pearson) &, $780^{* *}$ & 1 \\
& Signif. (bilateral) &, 000 & 28 \\
\hline
\end{tabular}

Nota. ${ }^{* *}$ La correlación es significativa en el nivel 0,01 (bilateral).

\section{Discusión}

$\mathrm{Al}$ aceptar la hipótesis general de investigación, se considera que existe relación entre la gestión educativa y el desempeño docente. Comparando las conclusiones propias son similares con los resultados de las investigaciones mencionadas. Consideramos que la propuesta teórica de Alvarado (2018) cuando explica que la gestión educativa es la aplicación práctica de un conjunto de técnicas, instrumentos y procedimientos, estas actividades se dimensionan en las actividades relacionadas con los alumnos, el uso de los recursos educativos y las actividades propias de la administración, de la cual se desprende la gestión pedagógica, institucional y administrativa. Y, que son adecuadas para la labor diaria del personal directivo que prioriza los propósitos y metas de aprendizaje en los estudiantes, sin descuidar los instrumentos de gestión y el buen uso de los recursos económicos, con los que cuenta una institución educativa.

Asimismo, Bush (2003) considera que la gestión educativa debe estar centrada en los aspectos pedagógicos. Este es un problema que ocurre en las diferentes escuelas públicas del Perú, donde las obligaciones administrativas que el Estado impone sobre la plana jerárquica de las instituciones educativas es excesiva, en especial con el mantenimiento preventivo, donde el director recibe fondos estatales para planificar, ejecutar y rendir balance de diversas obras de mantenimiento de infraestructura o compra de materiales, restándole tiempo valioso para dedicar a las diversas acciones de mejora pedagógica en el personal docente o el avance de los resultados académicos de los estudiantes.

Asimismo, referimos que es adecuada la propuesta de desempeño docente presentada por García et al. (2005) que divide la actividad del docente en las dimensiones: preparación y organización de actividades escolares, la conducción de las actividades de enseñanza, las relaciones pedagógicas porque permite la evaluación de los procesos de aprendizaje. Ello, permite entender la acción docente con el fin de lograr 
mejores aprendizajes en los estudiantes, evita la improvisación y presenta una secuencia lógica de actividades que aterrizan en la actividad del profesor en clase y la forma en que evalúa los aprendizajes.

La acción del director puede dimensionarse en ayudar al maestro a planificar mejor sus sesiones de aprendizaje bajo el enfoque del desarrollo de las competencias, identificar su conducta en la sesión misma de aprendizaje, el nivel de las relaciones pedagógicas en la clase, sobre la forma en que los estudiantes conviven en el aula y el fomento de la evaluación formativa en la acción docente. Se requiere que el personal jerárquico en las escuelas pueda identificar claramente los aspectos teóricos de la gestión educativa y el desempeńo docente, a fin de tener la claridad sobre su acción diaria en la gestión, de esta manera sus decisiones serán más acertadas para la importante labor que ejerce.

\section{Conclusiones}

Se concluye que los directores deben conocer el marco teórico de la gestión educativa a fin de tomar las decisiones más adecuadas en la dirección de una escuela, que dentro de sus funciones pedagógicas, institucionales y administrativas. La principal debe ser la pedagógica que mediante el liderazgo pedagógico debe asumir la responsabilidad de influenciar a sus profesores en las diversas acciones que están relacionadas con los procesos de enseñanza aprendizaje, desde la planificación, ejecución de clases, las relaciones humanas entre los alumnos y las formas de evaluación.

Adicionalmente, el Estado del Perú mediante el Ministerio de Educación estipula que el director realice funciones administrativas, las cuales disminuyen el tiempo y la dedicación de la plana jerárquica a realizar acciones eminentemente pedagógicas. También, mediante concursos públicos llamados encargaturas, se eligen directores a docentes sin ninguna experiencia en la gestión pública, por lo que es importante realizar acciones de capacitación y acompańamiento al director por parte del personal especializado de la unidad de gestión educativa local.

\section{Referencias}

Alvarado, O. (2018). Gestión Educativa. Editorial Universitaria.

Bush, T. (2003). Theories of Educational Leadership and Managemen [Teorias de la Administración y el Liderazgo en Educación]. SAGE Publications.

Cejas, A. (2009). Gestión Educativa. Revista Integra Educativa, 2(3), 215-231. http://www.scielo.org.bo/scielo. php?pid=S1997-40432009000300010\&script=sci_abstract

García, A., y Ferreira, G. (2005). La convivencia escolar en las Aulas. International Journal of Developmental and Educational Psychology, 2, 163-183

González et al. (2021). La Preparación de los Maestros para Estimular la Socialización De los Educandos con Autismo en Condiciones de Inclusión. Revista Brasileira de Educação Especial v. 27, e0197. 355-370. https://www.scielo. br/j/rbee/a/Q634NMSTTS85v7pwJJtbHVd/?lang=es\#

Hernández, R., Fernández, C., y Baptista, M. (2014). Metodología de la Investigación (6a ed.). Mc Graw-Hill.

López Rodríguez del Rey, María Magdalena, Fuentes Suárez, Iselys, \& Sánchez González, Katia. (2021). La preparación de los estudiantes para el proceso de evaluación y acreditación. Conrado, 17(79), 157-162. Epub 02 de abril de 2021. http://scielo.sld.cu/scielo.php?script=sci_arttext\&pid=S1990-86442021000200157\&lng $=$ es\&tlng=es.

Marín-González, Freddy, Grimaldo-Guerrero, John, Mendoza-Becerra, Manuel, \& Senior-Naveda, Alexa. (2020). Mapeo del conocimiento para la enseñanza interdisciplinaria en ingeniería. Formación universitaria, 13(6), 8594. https://dx.doi.org/10.4067/S0718-50062020000600085 
Martínez - Chairez, Guadalupe Iván, \& Guevara - Araiza, Albertico, \& Valles - Ornelas, María Manuela (2016). EL DESEMPEÑO DOCENTE Y LA CALIDAD EDUCATIVA. Ra Ximhai, 12(6),123-134. [fecha de Consulta 27 de octubre de 2021]. ISSN: 1665-0441. Disponible en: https://www.redalyc.org/articulo.oa?id=46148194007

Vásquez-Rodríguez, F. (2010). Estrategias de enseñanza: investigaciones sobre didáctica en instituciones educativas de la ciudad de Pasto. http://biblioteca.clacso.edu.ar/Colombia/fce-unisalle/20170117011106/Estrategias.pdf

\section{Notas al final}

1 Licenciado en Educación por la Universidad Nacional Mayor de San Marcos. Actualmente se desempeña como directivo en la Unidad de Gestión Educativa Local de la provincia de Recuay. Especialista en procesos de capacitación e implementación del Currículo Nacional en docentes de la localidad de Recuay y programas de gestión educativa para el personal directivo de educación básica. 